\title{
FORMULAS FOR THE JOINT SPECTRAL RADIUS OF NON-COMMUTING BANACH ALGEBRA ELEMENTS
}

\author{
P. ROSENTHAL AND A. SOLTYSIAK
}

(Communicated by Palle E. T. Jorgensen)

\begin{abstract}
We discuss relations between several formulas for the joint spectral radius of $n$-tuples of non-commuting Banach algebra elements.
\end{abstract}

In 1960, Rota and Strang [10] proposed a formula for the joint spectral radius of an $n$-tuple (or, more generally, any bounded set) of Banach algebra elements. A different formula was investigated by Berger and Wang [3]. We consider relations between these formulas and the "geometric spectral radius".

Let $\mathscr{A}$ be a unital complex Banach algebra, and let $\left(a_{1}, \ldots, a_{n}\right)$ be an $n$-tuple of elements of $\mathscr{A}$. Let $F(s, n)$ be the set of all functions from $\{1, \ldots, s\}$ to $\{1, \ldots, n\}$. Then the Rota-Strang radius $\hat{r}$ is defined by $\hat{r}\left(a_{1}, \ldots, a_{n}\right)=\lim _{s \rightarrow \infty} \max _{f \in F(s, n)}\left\|a_{f(1)} a_{f(2)} \cdots a_{f(s)}\right\|^{1 / s}$. (In [10] it is shown that this limit always exists.) The Berger-Wang radius $r_{*}$ is defined by

$$
r_{*}\left(a_{1}, \ldots, a_{n}\right)=\limsup _{s \rightarrow \infty} \max _{f \in F(s, n)}\left[r\left(a_{f(1)} a_{f(2)} \cdots a_{f(s)}\right)\right]^{1 / s},
$$

where $r$ is the ordinary spectral radius in $\mathscr{A}$.

The left joint spectrum $\sigma_{l}\left(a_{1}, \ldots, a_{n}\right)$ of an $n$-tuple of elements of $\mathscr{A}$ is the set of all $n$-tuples $\left(\lambda_{1}, \ldots, \lambda_{n}\right)$ of complex numbers for which the left ideal generated by $\left\{a_{1}-\lambda_{1}, a_{2}-\lambda_{2}, \ldots, a_{n}-\lambda_{n}\right\}$ is proper; the right joint spectrum is defined analogously. We denote by $\hat{\sigma}\left(a_{1}, \ldots, a_{n}\right)$ the union of the left and right joint spectra of $\left(a_{1}, \ldots, a_{n}\right)$, computed with respect to the unital algebra generated by $\left\{a_{1}, \ldots, a_{n}\right\}$ (cf. [8]). The geometric joint spectral radius $r\left(a_{1}, \ldots, a_{n}\right)$ of $\left(a_{1}, \ldots, a_{n}\right)$ is then defined by

$$
r\left(a_{1}, \ldots, a_{n}\right)=\max \left\{\left|\lambda_{j}\right|:\left(\lambda_{1}, \ldots, \lambda_{n}\right) \in \hat{\sigma}\left(a_{1}, \ldots, a_{n}\right)\right\} .
$$

That is, $r\left(a_{1}, \ldots, a_{n}\right)$ is the radius of the smallest $l^{\infty}$ ball in $\mathbb{C}^{n}$ containing $\hat{\sigma}\left(a_{1}, \ldots, a_{n}\right)$.

Easy examples [8] show that $\hat{\sigma}\left(a_{1}, \ldots, a_{n}\right)$ may be empty; in that case we put $r\left(a_{1}, \ldots, a_{n}\right)=-\infty$.

Theorem 1. For any $\left(a_{1}, \ldots, a_{n}\right)$,

$$
r\left(a_{1}, \ldots, a_{n}\right) \leq \max _{1 \leq j \leq n} r\left(a_{j}\right) \leq r_{*}\left(a_{1}, \ldots, a_{n}\right) \leq \hat{r}\left(a_{1}, \ldots, a_{n}\right) .
$$

Received by the editors January 10, 1994.

1991 Mathematics Subject Classification. Primary 46H05, 47A13.

(C) 1995 American Mathematical Society 
Proof. The only inequality that is not immediate is the first. For this, assume that $\left(\lambda_{1}, \ldots, \lambda_{n}\right) \in \hat{\sigma}\left(a_{1}, \ldots, a_{n}\right)$. Then, by [5], there is a multiplicative linear functional $\phi$ on the unital Banach $\mathscr{A}_{0}$ algebra generated by $\left\{a_{1}, \ldots, a_{n}\right\}$ such that $\phi\left(a_{i}\right)=\lambda_{j}$ for $j=1, \ldots, n$. Then $\phi\left(a_{j}\right)$ is in the spectrum of $a_{j}$ computed with respect to the algebra $\mathscr{A}_{0}$. Since the ordinary spectral radius of an element does not depend on the algebra containing it, the result follows.

Example. There exists $\left(a_{1}, a_{2}\right)$ with $r_{*}\left(a_{1}, a_{2}\right)=0$ and $\hat{r}\left(a_{1}, a_{2}\right)=1$.

Proof. We use an example constructed by Guinand [6] for a different purpose. Let $\mathscr{A}$ be the Banach algebra of all bounded linear operators on a separable complex Hilbert space. We construct two weighted shifts as follows. Let $\left\{\alpha_{j}\right\}$ be a sequence of 0 's and 1 's such that no finite substring occurs three times in a row; the existence of such a sequence was established by Thue ([12], Satz 6); also see ([7], Problem 1J). Choose an orthonormal basis $\left\{e_{j}\right\}_{j=0}^{\infty}$ and define

$$
T_{1} e_{j}=\alpha_{j} e_{j+1}, \quad T_{2} e_{j}=\left(1-\alpha_{j}\right) e_{j+1}
$$

for $j=0,1,2,3, \ldots$. Then each $T_{i}$ is a bounded linear operator, and every word in $\left\{T_{1}, T_{2}\right\}$ is nilpotent of order 3 (see [6]).

Since every word in $\left\{T_{1}, T_{2}\right\}$ is nilpotent, $r_{*}\left(T_{1}, T_{2}\right)=0$. But there exist arbitrarily long words in $\left\{T_{1}, T_{2}\right\}$ of norm 1: simply let

$$
f(j)= \begin{cases}1 & \text { if } \alpha_{j-1} \neq 0 \\ 2 & \text { if } \alpha_{j-1}=0\end{cases}
$$

for each $j$ and form

$$
W=T_{f(s)} T_{f(s-1)} \cdots T_{f(1)}
$$

Then $\left\|W e_{0}\right\|=1$, so $\|W\|=1$. Thus $\max _{f \in F(s, 2)}\left\|T_{f(1)} \cdots T_{f(n)}\right\|^{1 / n}=1$ for all $s$, and $\hat{r}\left(T_{1}, T_{2}\right)=1$.

Notes. 1. For finite-dimensional matrices $\left\{T_{i}\right\}$, it is shown in [3] that

$$
\hat{r}\left(T_{1}, \ldots, T_{n}\right)=r_{*}\left(T_{1}, \ldots, T_{n}\right) .
$$

2. Similarly, if the elements $\left\{a_{i}\right\}$ are mutually commuting, then it is shown in [11] that

$$
r\left(a_{1}, \ldots, a_{n}\right)=\hat{r}\left(a_{1}, \ldots, a_{n}\right),
$$

so by Theorem 1 it follows that $r_{*}\left(a_{1}, \ldots, a_{n}\right)=\hat{r}\left(a_{1}, \ldots, a_{n}\right)$ in that case too.

Let $\operatorname{rad} \mathscr{A}$ denote the Jacobson radical of $\mathscr{A}$.

Theorem 2. If $\mathscr{A}$ is a unital complex Banach algebra, then the following are equivalent:

(i) $\mathscr{A} / \operatorname{rad} \mathscr{A}$ is commutative;

(ii) $r\left(a_{1}, \ldots, a_{n}\right)=\max \left\{r\left(a_{j}\right): j=1, \ldots, n\right\}$ for every finite subset $\left\{a_{1}, \ldots, a_{n}\right\}$ of $\mathscr{A}$

(ii') $r\left(a_{1}, a_{2}\right)=\max \left\{r\left(a_{1}\right), r\left(a_{2}\right)\right\}$ for each pair $\left\{a_{1}, a_{2}\right\} \subset \mathscr{A}$;

(iii) for every $n, r\left(a_{1}, \ldots, a_{n}\right)=r_{*}\left(a_{1}, \ldots, a_{n}\right)$ for all $n$-tuples of elements of $\mathscr{A}$;

(iii') $\quad r\left(a_{1}, a_{2}\right)=r_{*}\left(a_{1}, a_{2}\right)$ for each pair $\left\{a_{1}, a_{2}\right\} \subset \mathscr{A}$. 
Proof. That (ii) implies (ii') and that (iii) implies (iii') are trivial. By Theorem 1, (iii) implies (ii) and ( iii $\left.^{\prime}\right)$ implies $\left(\right.$ ii $\left.^{\prime}\right)$.

To show that (ii') implies (i), let $a$ and $b$ be arbitrary elements of $\mathscr{A}$. By the definitions and the fact that the unital subalgebras generated by $\{a, b\}$ and $\{a+b, a\}$ are identical,

$$
r(a+b, a)=\max \{|\alpha|:(\alpha, \beta) \text { or }(\beta, \alpha) \text { is in } \hat{\sigma}(a+b, a)\} .
$$

Thus, in particular, $\hat{\sigma}(a+b, a) \neq \varnothing$. By [5], there is a unital multiplicative linear functional $\phi$ on the unital algebra generated by $\{a, b\}$ such that

$$
r(a+b, a)=\max \{|\phi(a+b)|,|\phi(a)|\} .
$$

Thus $r(a+b, a) \leq|\phi(a)|+|\phi(b)|$.

But clearly $|\phi(a)| \leq r(a)$ and $|\phi(b)| \leq r(b)$, since the spectral radius of a single element is independent of the Banach algebra containing it. Thus, $r(a+b, a) \leq r(a)+r(b)$. But $r(a+b) \leq r(a+b, a)$ by $\left(\right.$ ii $\left.^{\prime}\right)$, so we have

$$
r(a+b) \leq r(a)+r(b) \text {. }
$$

The Aupetit-Zemanek Theorem ([1], [13], or [2, p. 94]) states that such subadditivity of the spectral radius implies that $\mathscr{A} / \operatorname{rad} \mathscr{A}$ is commutative, so (ii') implies (i).

It remains to be shown that (i) implies (iii). For an element $a \in \mathscr{A}$, let [a] denote its coset in $\mathscr{A} / \operatorname{rad} \mathscr{A}$. Then $r(a)=r([a])$ for every $a \in \mathscr{A}$, so

$$
r_{*}\left(\left[a_{1}\right], \ldots,\left[a_{n}\right]\right)=r_{*}\left(a_{1}, \ldots, a_{n}\right) .
$$

But $\hat{\sigma}\left(\left[a_{1}\right],\left[a_{2}\right], \ldots,\left[a_{n}\right]\right) \subset \hat{\sigma}\left(a_{1}, \ldots, a_{n}\right)$ (from the definitions), so

$$
r\left(\left[a_{1}\right],\left[a_{2}\right], \ldots,\left[a_{n}\right]\right) \leq r\left(a_{1}, \ldots, a_{n}\right) .
$$

Since $\mathscr{A} / \operatorname{rad} \mathscr{A}$ is commutative,

$$
r\left(\left[a_{1}\right],\left[a_{2}\right], \ldots,\left[a_{n}\right]\right)=r_{*}\left(\left[a_{1}\right],\left[a_{2}\right], \ldots,\left[a_{n}\right]\right) .
$$

Thus

$$
\begin{aligned}
r_{*}\left(a_{1}, a_{2}, \ldots, a_{n}\right) & =r_{*}\left(\left[a_{1}\right],\left[a_{2}\right], \ldots,\left[a_{n}\right]\right) \\
& =r\left(\left[a_{1}\right],\left[a_{2}\right], \ldots,\left[a_{n}\right]\right) \\
& \leq r\left(a_{1}, a_{2}, \ldots, a_{n}\right) .
\end{aligned}
$$

But, by Theorem 1, $r\left(a_{1}, a_{2}, \ldots, a_{n}\right) \leq r_{*}\left(a_{1}, a_{2}, \ldots, a_{n}\right)$, so (iii) holds.

Note. By Theorems 1 and $2, r\left(a_{1}, a_{2}, \ldots, a_{n}\right)=\hat{r}\left(a_{1}, a_{2}, \ldots, a_{n}\right)$ implies each of the conditions of Theorem 2 . We do not know, however, if the converse holds. Equivalently, if $\mathscr{A}$ is commutative modulo its radical, must

$$
r_{*}\left(a_{1}, a_{2}, \ldots, a_{n}\right)=\hat{r}\left(a_{1}, a_{2}, \ldots, a_{n}\right)
$$

for all $n$-tuples of elements of $\mathscr{A}$ ?

\section{REFERENCES}

1. B. Aupetit, Caractérisation spectrale des algébres de Banach commutatives, Pacific J. Math. 63 (1976), 23-35.

2. __ A primer on spectral theory, Universitext, Springer-Verlag, New York, 1991. 
3. M. A. Berger and Y. Wang, Bounded semigroups of matrices, Linear Algebra Appl. 166 (1992), 21-27.

4. M. Chō and Żelazko, On geometric spectral radius of commuting $n$-tuples of operators, Hokkaido Math. J. 21 (1992), 251-258.

5. C. K. Fong and A. Soltysiak, Existence of a multiplicative linear functional and joint spectra, Studia Math. 81 (1985), 213-220.

6. P. S. Guinand, On quasinilpotent semigroups of operators, Proc. Amer. Math. Soc. 86 (1982), 485-486.

7. P. R. Halmos, Problems for mathematicians young and old, Math. Assoc. Amer., Washington, DC, 1991.

8. R. E. Harte, Spectral mapping theorems, Proc. Roy. Irish Acad. Sect. A 72 (1972), 89-107.

9. V. Müller and A. Soltysiak, Spectrum of generators of a noncommutative Banach algebra, Studia Math. 93 (1989), 87-95.

10. G.-C. Rota and W. G. Strang, A note on the joint spectral radius, Indag. Math. 22 (1960), 379-381.

11. A. Soltysiak, On the joint spectral radii of commuting Banach algebra elements, Studia Math. 105 (1993), 93-99.

12. A. Thue, Ueber unendliche Zeichenreihen, Selected Mathematical Papers of Axel Thue, Universitetsforlaget, Oslo-Bergen-Tromsø, 1977, pp. 139-158.

13. J. Zemánek, Spectral radius characterization of commutativity in Banach algebras, Studia Math. 61 (1977), 257-268.

Department of Mathematics, University of Toronto, Toronto, Ontario, Canada M5S $1 \mathrm{~A} 1$

E-mail address: rosent@math.toronto.edu

Faculty of Mathematics and Computer Science, A. Mickiewicz University, Matejki 48/49, 60-769 Poznań, Poland

E-mail address: asoltys@plpuam11.bitnet 